\title{
Effects of semantic context and expectancy in a lexical decision and naming task
}

\author{
REGINA McGLINCHEY-BERROTH and WILLIAM P. MILBERG \\ V.A. Medical Center, West Roxbury, Massachusetts
}

\begin{abstract}
Reported differences in the sensitivity of the lexical decision and naming tasks to postlexical processes was investigated in a single-word priming study. Relatedness probability was manipulated with an "induction set" presented to subjects prior to the onset of the actual test stimuli. In the naming task, exposure to an induction set with a high probability of related word pairs produced both facilitation and inhibition, whereas exposure to an induction set with a high probability of unrelated word pairs resulted in similar response times to all word targets. Significant facilitation was observed in the lexical decision task, regardless of the relatedness probability of the induction set. These data suggest that under certain circumstances the naming task is sensitive to postrecognition contextual effects, and they do not support the use of the lexical decision and naming tasks as methodological tools with which to a priori distinguish pre- and postcontextual effects in word recognition.
\end{abstract}

A consensus seems to be emerging in the literature on lexical context effects that lexical decision and word pronunciation (naming) tasks gauge different aspects of processes involved in word recognition. Although both lexical decision and naming responses appear to be sensitive to the prelexical process of spreading activation, evidenced by equally large facilitory effects when target words are preceded by semantically related words (in both wordlist and sentence paradigms), lexical decision responses appear to be more affected by experimental variables that presumably can have their impact only on postlexical word recognition (DeGroot, 1985; Seidenberg, Waters, Sanders $\&$ Langer, 1984). However, inherent differences in the way these tasks were designed in previous studies make this interpretation problematic.

The present study also investigates contextual effects in word recognition and seeks to disambiguate findings from the studies mentioned above. Many differences exist between the present study and those previously outlined. First, a technique designed to maximize the effects of postlexical integrative influences on processing was used. An "induction set" of word pairs presented prior to the onset of the actual test stimuli manipulated the probability of related word pairs (S. Blumstein, personal communication, December 1985). In the "high-probability" induction set, all real-word pairs were related. In the "low-probability" induction set, all real-word pairs were unrelated. In this way, the probability of related and unrelated word targets was

This research was supported by V.A. Merit Review 097-44-3765001 and NIA Grant RO 1 AG 033540-3 to William Milberg. The authors thank David Swinney, Richard Chechile, and Patrick Kilduff for their comments on an earlier version of this manuscript and Anna Barrett and Laura Grande for their assistance throughout this project. Correspondence should be addressed to R. McGlinchey-Berroth, Geriatric Research, Education and Clinical Center, Brocton/West Roxbury V.A. Medical Center (182), 1400 VFW Parkway, West Roxbury, MA 02132. identical for all subjects within the actual test stimuli. Using an induction set also increases the likelihood that the probability manipulation will effect response latencies because it maximizes strategies prior to the onset of the actual test trials. Response latencies, then, reflect an established straegy rather than a developing one. Secondly, the test stimuli are identical for the naming and lexical decision tasks. In this way the probability that a target item is preceded by a related, unrelated, or nonword prime item is identical across the two types of response tasks. Lastly, contextual effects are measured relative to a nonword prime/word target condition. The use of nonword primes with word targets to form the baseline condition was both theoretically and empirically based. First, various other types of primes that are typically used (e.g., XXXX, BLANK, THE, READY) have disadvantages that may render them inappropriate for use as a baseline measure. One obvious disadvantage is that these primes are repeated many times more than other, nonneutral primes. This can potentially result in a differential employment of attention across these conditions. Empirically, a number of previous studies have used this method, and it has been found to be an effective means to discourage the development of predictive strategies (e.g., Blumstein, Milberg, \& Shrier, 1982; Milberg and Blumstein, 1981; Milberg, Blumstein, \& Dworetzky, 1987, 1988).

\section{METHOD}

\section{Subjects}

Forty-eight subjects participated in this study; they varied in age from 26 to 61 years (mean $=56$ years). All subjects reported their vision as normal or corrected-to-normal. They were all native English-speaking volunteers from the Veterans Administration Medical Center, West Roxbury, MA.

\section{Stimuli \\ The stimuli used in this study were composed of word and nonword pairs. The first letter string of a pair was considered the prime, and} the second letter string of a pair was considered the target. Four lists 
of prime-target pairs were prepared. Two lists of 48 trials made up the related and unrelated induction sets. The remaining two stimulus lists, containing 96 trials each, made up the test stimuli.

The induction sets contained three types of prime-target pairs. Twentyfour target words were preceded by real-word primes. In the related induction list, all word primes were highly associated with the target words. In the unrelated induction list, the target words were paired with unassociated real-word primes. Eight additional word targets were preceded by pronounceable nonword primes. In addition there were 16 pronounceable nonword target items; 8 were preceded by word primes, and 8 were preceded by nonword primes.

Lists A and B, which made up the test stimuli, contained five types of prime-target pairs. Three pairs had word targets and were considered the critical stimuli. In both Lists A and B, 16 target words were preceded by highly associated word primes (e.g., dog-cat), 16 were preceded by unassociated word primes (e.g., hand-night), and 16 were preceded by pronounceable nonword primes (e.g., blint-cereal). The unrelated target pairs were formed by randomly selecting 8 related target words from each list and re-pairing them with unassociated novel prime words. For example, in constructing the unrelated pairs for List A, 8 related targets from List $A$ and 8 related targets from List B were re-paired with unrelated primes to form the 16 unrelated trials for List $A$. The remaining trials from both lists were re-paired with unassociated primes to form the unrelated trials in List B. A similar procedure was used in constructing the nonword prime/word target trials. For List $A$, the 8 remaining target words that were not repeated in the unrelated condition in List $A$ and the 8 remaining targets that were not selected from list B were re-paired with pronounceable nonword primes for List $A$. The same procedure was used in constructing the nonword prime trials for List $\mathrm{B}$.

The last two types of prime-target pairs were composed of pronounceable nonword targets. Twenty-four nonword targets were preceded by word primes (e.g., boot-slunk), and 24 were preceded by nonword primes (e.g., thorch-groven)

It should be noted that given this design, the probability of a realword target $(p=.57)$ was slightly higher than that of a nonword target $(p=.43)$, given a real-word prime. Such a design would compromise the predictions about priming effects only by making the tasks more conservative. Even if this slight difference in probability can be detected by the subjects, the knowledge that a real-word target was more likely following a real-word prime would only serve to proportionally decrease the response latency for targets preceded by both related and unrelated primes. This result may truncate the difference between these conditions but would not undermine claims about the presence of priming or, to an even greater degree, inhibition.

\section{Apparatus}

The stimulus word pairs were presented via an Apple IIe microcomputer. The computer was equipped with a Mountain Computer clock to control stimulus presentation and measure response latencies. Two telegraph keys mounted on a board with an attached microphone were interfaced with the computer. In the lexical decision task, depression of either telegraph key stopped the millisecond timer. In the naming task, a vocal response into the microphone stopped the timer. The sensitivity of the voice-operated relay was adjusted for each subject prior to the testing session.

\section{Procedure}

The subjects were tested individually in a session lasting approximately $40 \mathrm{~min}$. They were assigned to one of eight conditions according to their arrival at the testing session. The eight conditions were formed by crossing the two versions of the test stimuli $\times$ induction set $\times$ response task Within each of these conditions, the subjects were presented the 48 trials of the induction set followed by the 96 test trials.

Three asterisks centered on the video display monitor signaled the onset of each trial. The asterisks were displayed for $1 \mathrm{sec}$ and were then replaced by the prime letter string, centered where the asterisks had been. The prime was presented for $\mathbf{4 0 0} \mathrm{msec}$. Following an interstimulus interval (ISI) of $\mathbf{4 0 0 ~ m s e c , ~ t h e ~ t a r g e t ~ s t r i n g ~ w a s ~ d i s p l a y e d ~ w i t h i n ~ a ~ c e n - ~}$ tered, $1 \frac{1}{2} \times 3$ in. rectangular box. The target letter string remained displayed on the video display monitor until a response was made by the subject. Response latencies were recorded by the computer from target onset to the subjects' response. Correct/incorrect feedback was provided automatically by the computer: a high pitched tone signified a correct response, and a low pitched tone signified an incorrect response.

Each subject was told that two strings of letters that might or might not spell a real English word would appear on the screen, one after the other. He was instructed to read the first string silently and to respond only to the second string. When making lexical decisions, he was instructed to depress the marked "YES" key with the index finger of his dominant hand if the letter string spelled a word and to depress the marked "NO" key if it did not spell a real word. Prior to responding, the subject rested his index finger on the board midway between the two telegraph keys. When naming, the subject was instructed to read the target string aloud into the hand-held microphone whether or not the string spelled a real word. The subject was encouraged to respond as quickly and as accurately as possible.

\section{RESULTS}

\section{Preliminary Analyses}

The subjects' mean correct response times to target words from Stimulus Lists A and B were compared. The mean latency of $904 \mathrm{msec}$ for List A did not differ significantly from the mean latency of $883 \mathrm{msec}$ for List B in an unpaired $t$ test $(p>.05)$. The two lists were thus collapsed to form one data set for the remaining analyses.

To determine if the slightly higher probability for word targets following word primes biased the subjects to respond on the basis of the lexical status of the prime, the number of errors was broken out into four conditions: (1) word prime/nonword target, (2) nonword prime/word target, (3) word prime/word target, and (4) nonword prime/nonword target. It was hypothesized that if the subjects did, in fact, use the nature of the priming stimulus to predict the nature of the target, more errors would have occurred in Conditions 1 and 2 than in Conditions 3 and 4. The pattern of errors, however, did not support this possibility ( 9 total errors in Conditions 1 and 2; 13 total errors in Conditions 3 and 4).

\section{General Analysis}

For each subject, response times to target words were trimmed to within two standard deviations of their overall mean. On this basis, $4 \%$ of all trials were excluded, averaging two trials per subject. Table 1 shows the trimmed, mean response times and corresponding standard deviations for correct responses broken down by response task, induction set, and prime type. Table 1 also displays the number of errors observed in these conditions. It can be seen that the error rate was negligible, and thus it was not examined statistically. It is also important to note that the average response time for target words preceded by nonword primes fell between that for targets preceded by both related and unrelated primes in all but one condition. These times are thus assumed to represent a functionally neutral baseline of lexical retrieval from which facilitory and inhibitory effects may be estimated.

Responses to nonword target items is not directly relevant to the issues under study and were therefore not examined statistically. However, Table 2 displays the mean response time, standard deviation, and error rate for nonword target conditions. The between-subject effects of response task (lexical decision, naming) and induction set 
(related, unrelated) and the within-subject effect of prime type (related, unrelated, nonword) on the subjects' response times to target words were examined in a mixed three-way ANOVA. This ANOVA revealed a significant effect of response type $[F(1,44)=4.03, p<.05]$. Consistent with previous investigations, naming responses averaged $136 \mathrm{msec}$ shorter than lexical decision responses.

The type of prime also affected responses to target words, $[F(2,88)=19.93, p<.001]$. Analysis of simple effects (Keppel, 1982) indicated that responses to targets preceded by related primes were faster than to targets preceded by nonword primes $[F(1,88)=5.26$, $p<.02]$. The unrelated primes resulted in longer latencies to target words than nonword primes $[F(1,88)=$ $9.43, p<.01]$.

A significant prime type $\times$ induction set interaction, $[F(2,88)=5.22, p<.01]$ indicated that the effect of prime type differed as a function of induction set. A post hoc analysis was conducted with the Neuman-Keuls test (Winer, 1971) to examine the priming effects within the related and unrelated induction sets. Given a related induction set, response times with target words were faster when preceded by related primes compared with targets preceded by unrelated $(p<.01)$ and nonword $(p<.01)$ primes. In addition, the subjects exposed to the related induction set responded more slowly to target words preceded by unrelated word primes compared with those preceded by nonword primes $(p<.05)$. No comparisons involving the unrelated induction set reached significance.

Although the means displayed in Table 1 are suggestive of a three-way interaction of response type, induction set, and prime type, it was not found to be significant in this

Table 1

Trimmed Mean Correct Response Times (in Milliseconds), Standard Deviations, and Errors for Word Targets

\begin{tabular}{|c|c|c|c|c|c|c|c|}
\hline \multirow[b]{3}{*}{ Task } & \multirow[b]{3}{*}{ Prime Type } & \multicolumn{6}{|c|}{ Induction Set } \\
\hline & & \multicolumn{3}{|c|}{ Related } & \multicolumn{3}{|c|}{ Unrelated } \\
\hline & & RT & $S D$ & ER & RT & $S D$ & $\overline{E R}$ \\
\hline \multirow[t]{3}{*}{ Naming } & related & 772 & 187 & 1 & 797 & 253 & 0 \\
\hline & unrelated & 953 & 379 & 0 & 820 & 216 & 0 \\
\hline & nonword & 888 & 287 & 0 & 817 & 221 & 0 \\
\hline \multirow{3}{*}{$\begin{array}{l}\text { Lexical } \\
\text { decision }\end{array}$} & related & 909 & 205 & 4 & 939 & 313 & 0 \\
\hline & unrelated & 1,041 & 213 & 1 & 1,011 & 284 & 1 \\
\hline & nonword & 995 & 215 & 0 & 1,026 & 295 & 2 \\
\hline
\end{tabular}

Note-RT $=$ response time; ER $=$ number of errors.

Table 2

Mean Response Times (in Milliseconds) and Standard Deviations for Nonword Targets

\begin{tabular}{cccccc}
\hline \multirow{2}{*}{ Task } & Prime Type & RT & $S D$ & & \multicolumn{3}{c}{ Induction } & Set \\
\cline { 3 - 6 } \cline { 3 - 6 } Naming & word & 1,157 & 540 & & \multicolumn{2}{c}{ Related } & & Unrelated \\
\cline { 3 - 6 } & nonword & 1,187 & 523 & & 631 \\
& & & & & 626 \\
Lexical & word & 1,101 & 341 & 1,188 & 448 \\
decision & nonword & 1,130 & 373 & 1,178 & 447 \\
\hline
\end{tabular}

Table 3

Mean Facilitory and Inhibitory Effects (in Milliseconds) of Primes and Corresponding $t$ Values

\begin{tabular}{|c|c|c|c|c|c|}
\hline \multirow[b]{3}{*}{ Task } & \multirow[b]{3}{*}{ Prime Type } & \multicolumn{4}{|c|}{ Induction Set } \\
\hline & & \multicolumn{2}{|c|}{ Related } & \multicolumn{2}{|c|}{ Unrelated } \\
\hline & & $\mathrm{RT}$ & $t$ Value & RT & $t$ Value \\
\hline \multirow[t]{2}{*}{ Naming } & faciliation & 116 & $2.80 \dagger$ & 20 & 0.93 \\
\hline & inhibition & 64 & $1.93^{*}$ & 2 & 0.43 \\
\hline \multirow{2}{*}{$\begin{array}{l}\text { Lexical } \\
\text { decision }\end{array}$} & faciliation & 86 & $3.62 \dagger$ & 87 & $3.29 \dagger$ \\
\hline & inhibition & 46 & 1.33 & -15 & 1.38 \\
\hline
\end{tabular}

ANOVA $[F(2,88)=1.37]$. The main effect of induction set $[F(2,44)=0.11]$ and the interaction between response type and prime type $[F(2,88)=0.19]$ were also nonsignificant.

\section{Analysis of Facilitation and Inhibition}

A series of $t$ tests was conducted to examine specifically the presence of facilitation and inhibition within the two induction sets and response types. The amount of facilitation was derived by subtracting the subjects' mean response latency to target words following related word primes from that for target words following nonword primes. The amount of inhibition was derived by subtracting the subjects' mean response latency to target words following nonword primes from the subjects' mean response latency to target words following unrelated primes. The difference scores were then tested to determine if they deviated significantly from zero.

Table 3 displays the mean facilitation and inhibition observed within the two induction sets as a function of response type, as well as the obtained $t$ values. As suggested in the ANOVA, the amount of facilitation was significant in the lexical decision task for the subjects exposed to either the related or unrelated induction set. The mean difference in facilitation of $1.27 \mathrm{msec}$ between the related and unrelated induction set was not significant $(t<1)$ for lexical decisions. In contrast, the amount of facilitation in naming responses was significant only for the subjects who were exposed to the related induction set. Furthermore, the mean difference in facilitation of $95.60 \mathrm{msec}$ between the related and unrelated induction sets for naming responses was significant $[t(22)=2.02, p<.05$, one tailed], suggesting that naming responses were differentially influenced by induction set. Table 2 also shows that inhibition was observed only in naming responses for the subjects in the related induction condition. All other differences regarding inhibition did not exceed zero.

\section{DISCUSSION}

The present investigation sought to replicate previous reports indicating that the lexical decision and naming tasks are differentially sensitive to postlexical-meaning integration processes. Naming responses were considerably faster than lexical decision responses. This finding is consistent with a number of previous investigations (e.g., DeGroot, 1985; Forster \& Chambers, 1973; Seidenberg, et al., 1984). That naming responses are faster than lexical decision responses reflects the relative complexity of making lexical status judgments to target items compared with 
the pronunciation of target items. As suggested by Forster (1979), the locus of this complexity effect is at the postlexical level of processing, where the lexical status judgment is made, assuming that the two tasks share the same component processes of lexical access.

The effectiveness of an induction set to elicit differential processing of target words was evidenced by the presence of a prime type $X$ induction set interaction. Analysis of the interaction indicated that prior exposure to the related induction set (high relatedness probability) produced faster responses to target words preceded by related word primes compared with target words preceded by unrelated and nonword primes during later testing. Exposure to the related word induction set also resulted in longer response times to target words that were unrelated to the preceding prime word compared with target words preceded by nonword primes. Conversely, prior exposure to an unrelated induction set (low relatedness probability) effectively minimized the beneficial effects of related word primes and the detrimental effects of unrelated word primes in responding to target words.

Taken alone, these findings suggest that both the lexical decision task and the naming task are influenced by relatedness probability. However, different patterns emerged in the two tasks when facilitory and inhibitory effects were examined specifically. Naming responses were facilitated when targets were preceded by related word primes and inhibited when preceded by unrelated word primes (relative to nonword primes) for the subjects exposed to the high-probability induction set. No facilitory or inhibitory effects were observed for the subjects in the naming task who were exposed to an unrelated induction set. This suggests that in word naming, the inability to predict probable target items reduces the extent of inhibition, thus making the unrelated target words available for report more quickly. This expectancy also appears to decrease the amount of time required to pronounce target words preceded by nonword primes. As this condition was used as the baseline measure in which to compute facilitation, it is suggested that the apparent reduction in decision latency in the nonword prime condition obscured the facilitating effect of spreading activation that occurs for related word targets by truncating the difference between the two conditions. In the lexical decision task, facilitation was observed regardless of whether the subjects were exposed to the related or unrelated induction set. No significant inhibition was observed in the lexical decision task, probably because of the high level of variability in the data that resulted from the wide difference in the age of the subjects tested. Table 2 indicates that lexical decisions for unrelated target items in the high-probability condition averaged $46 \mathrm{msec}$ longer than decisions for neutrally primed targets. Given a more homogeneous sample, this difference would probably have been found to be significant.

Somewhat more disturbing is the lack of an overall relatedness probability effect in the lexical decision task. On the basis of previous studies, it was expected that the amount of facilitation would be greater in the related induction condition than in the unrelated induction condition. However, the effect was virtually identical: $86 \mathrm{msec}$ in the related condition and $87 \mathrm{msec}$ in the unrelated condition. A polynomial regression analysis was carried out to track facilitory and inhibitory effects within each induction set and response type. For this analysis, each subject's response times to target words for the three priming conditions were paired in the order of their presentation in the experiment, and the amount of facilitation and inhibition were computed on a trial-by-trial basis. For example, the response time for the first occurrence of a related word target was subtracted from the response time of the first occurrence of a target primed with a nonword to determine the amount of facilitation at Position 1 . Inhibitory effects were determined similarly by subtracting response times from nonword primed targets from response times from unrelated primed targets. Thus, facilitory and inhibitory effects were computed for 16 presentation positions. The polynomial regression analysis indicated that the magnitude of the facilitation effect in the unrelated induction condition grew throughout the course of the experiment. Thus, as the relative probability of related word pairs increased, so did the amount of facilitation. Perhaps the process of meaning integration becomes and remains a factor in the facilitation of target words once a critical proportion of related to unrelated (or neutral) pairs is presented. Other studies that have manipulated relatedness probability have done so by varying the proportion of related to unrelated trials within the experiment proper. As a result, the "critical" proportion may be reached early in these experiments, and then decline throughout the course of the trials sufficiently to obscure the original facilitative effect.
In this case, as the relative probability of related words decreases, so does the amount of facilitation. Thus, no facilitation is observed overall.

The pattern of results from the naming task is suggestive of postlexical influences, as considerable facilitation was found in the related induction condition with little or no facilitation in the unrelated condition. The regression analysis indicated that the amount of facilitation remained stable throughout the experiment. Similar to DeGroot (1985), it is suggested that the relatively long SOA of $800 \mathrm{msec}$ was effective in inducing pretarget recognition, context-induced attentional processes in the related induction condition. It is apparent, however, that meaning integration was also a factor in naming responses. A significant inhibition effect was found in the related induction condition for naming. Furthermore, the facilitation effect in that condition was considerably larger $(116 \mathrm{msec})$ than that found in the lexical decision task. It appears that the effects of meaning integration and context-induced attention processes are additive and may have contributed to the facilitation effect separately.

At first glance, this finding is somewhat curious in light of the fact that in a similar study, Seidenberg et al. (1984) did not find a difference in facilitation for naming responses. A close examination of the parameters of their study, however, reveals a possible explanation. Recall that Seidenberg et al. manipulated relatedness probability by varying the number of unrelated word pairs and holding the number of related word pairs constant across the high- and low-proportion conditions. In the naming task, there were 40 unrelated word pairs in the highproportion condition and 80 unrelated word pairs in the low-proportion condition. Response time to 20 "critical" unrelated target words was used as a baseline from which to measure facilitation. Given such a large number of unrelated pairs, it is possible that the response times to unrelated targets decreased and the response times to related targets increased, thereby reducing the magnitude of the difference in response times between the two types of prime-target pairs. In other words, the number of unrelated word pairs in the naming task may have been high enough, even in the high relatedness probability condition, to obscure a differential effect of the probability of related word pairs.

Additional research is needed that will focus specifically on the time course of probability effects in experiments of this type. Clearly, the contribution of pre- and postlexical processes in naming and lexical decision tasks is complex and warrants continued examination. The induction-set procedure used in this study provides a means by which contextual effects may be examined without potential confounds of "filler" targets and relatedness probability.

\section{REFERENCES}

Blumstein, S., Milberg, W. \& Shrier, R. (1982). Semantic processing and aphasia: Evidence from an auditory lexical decision task. Brain \& Language, 17, 301-315.

DeGroot, A. M. B. (1985). Word-context effects in word naming and lexical decision. Quarterly Journal of Experimental Psychology, 37A, 281-297.

FORSTER, K. I. (1979). Levels of processing and the structure of the language processor. In W. E. Cooper \& E. C. T. Walker (Eds.), Sentence processing: Psycholinguistic studies presented to Merrill Garrett (pp. 27-85). Cambridge: MIT Press.

Forster, K. I., \& ChAmbers, S. M. (1973). Lexical access and naming time. Journal of Verbal Learning \& Verbal Behavior, 12, 627-635.

KEPPEL, G. (1982). Design and analysis: A researcher's handbook. Englewood Cliffs, NJ: Prentice-Hall.

MilberG, W., \& BLUMSTEIN, S. (1981). Lexical decision and aphasia: Evidence for semantic processing. Brain \& Language, 14, 371-385.

Milberg, W., Blumstein, S. E., \& Dworetzky, B. (1987). Processing of lexical ambiguities in aphasia. Brain \& Language, 31, 138-150.

Milberg, W., Blumstein, S. E., \& Dworetzky, B. (1988). Phonological processing and lexical access in aphasia. Brain \& Language, 34, 279-293.

Seidenberg, M. S., Waters, G. S., SAnders, M., \& Langer, P. (1984). Pre- and postlexical loci of contextual effects on word recognition. Memory \& Cognition, 12, 315-328.

WINER, B. J. (1971). Statistical principles in experimental design. New York: McGraw-Hill.

(Manuscript received June 10, 1992.) 